\title{
The Role of von Willebrand Factor and Fibrinogen in Platelet Aggregation under Varying Shear Stress
}

\author{
Yasuo Ikeda, ${ }^{*}$ Makoto Handa, ${ }^{*}$ Koichi Kawano," Tetsuji Kamata, ${ }^{*}$ Mitsuru Murata, ${ }^{*}$ Yohko Araki, ${ }^{*}$ Hironobu Anbo, \\ Yohko Kawai," Kiyoaki Watanabe," Ichiro Itagaki," Kiyotaka Sakai," and Zaverio M. Ruggeri" \\ ${ }^{*}$ Blood Center, Departments of Internal Medicine and Laboratory Medicine, Keio University Hospital, Tokyo, Japan; ${ }^{\ddagger}$ Toray Industries \\ Inc., Kanagawa, Japan; 'Department of Chemical Engineering, Waseda University, Tokyo, Japan; and "Roon Research Laboratory for \\ Arteriosclerosis and Thrombosis, Department of Molecular and Experimental Medicine and Committee for Vascular Biology, \\ Scripps Clinic and Research Foundation, La Jolla, California 92037
}

\begin{abstract}
Exposure of platelets to shear stress leads to aggregation in the absence of exogenous agonists. We have now found that different adhesive proteins and platelet membrane glycoproteins are involved in aggregation depending on the shear stress conditions and the concentration of divalent cations in the medium. When blood is collected with trisodium citrate as anticoagulant, which causes a decrease in the levels of external ionized calcium $\left(\left[\mathrm{Ca}^{2+} \mathrm{b}\right)\right.$, platelet aggregation can be induced under low shear force $\left(12 \mathrm{dyn} / \mathrm{cm}^{2}\right)$ and is mediated by fibrinogen binding to the glycoprotein IIb-IIIa complex. Aggregates formed under these conditions are not stable, and when shear force is increased to $68 \mathrm{dyn} / \mathrm{cm}^{2}$, disaggregation results. By contrast, platelets from blood collected with hirudin as anticoagulant, wherein $\left[\mathrm{Ca}^{2+}\right]_{0}$ is within normal plasma levels, do not undergo low shear-induced aggregation; however, after exposure to a shear force above $80 \mathrm{dyn} / \mathrm{cm}^{2}$, aggregation is observed but only when von Willebrand factor is present and can interact with both its platelet binding sites, glycoprotein Ib-IX and glycoprotein IIb-IIIa. Fibrinogen is not involved in high shear-induced aggregation which, in fact, occurs normally in patients with severe afibrinogenemia. Thus, von Willebrand factor in the absence of exogenous agonists can mediate platelet aggregation in experimental conditions that may mimic the hemorheological situation of partially occluded arteries. This pathway of platelet aggregation involving only one adhesive ligand and two membrane adhesion receptors may play a relevant role in thrombogenesis. (J. Clin. Invest. 1991.87:1234-1240.) Key words: cell adhesion • thrombus formation • vascular occlusion
\end{abstract}

\section{Introduction}

The formation of platelet aggregates (thrombi) at sites of vascular injury is a normal response in the course of hemostasis and leads to the arrest of bleeding $(1,2)$. In disease states, however,

Parts of this work were presented at the Annual Meeting of the American Society for Clinical Investigation, Washington, D.C., 4-7 May 1990, and have been published in abstract form (1990. Clin. Res. 38:462A).

Address reprint requests to Dr. Zaverio M. Ruggeri, BCR-8, Scripps Clinic and Research Foundation, 10666 No. Torrey Pines Road, La Jolla, CA 92037.

Received for publication 21 August 1990 and in revised form 29 November 1990.

J. Clin. Invest.

(c) The American Society for Clinical Investigation, Inc. 0021-9738/91/04/1234/07 \$2.00

Volume 87, April 1991, 1234-1240 platelet aggregation may underlie the pathology associated with vascular occlusion (3-7). During thrombogenesis, circulating platelets become localized on altered vascular surfaces or exposed tissues (reviewed in 8 ) and become activated through a complex series of biochemical events (reviewed in 9). Activated platelets, then, adhere and aggregate to one another forming the thrombus mass, a process that has an absolute requirement for the membrane glycoprotein (GP) ${ }^{1}$ IIb-IIIa complex (reviewed in 10). Different experimental models have been used to investigate the molecular mechanisms of platelet adhesion and aggregation. Such models, however, reproduce only partially the complex series of events occurring during thrombogenesis in the intact organism; consequently, the results obtained may be limited in their significance by the specific set of conditions prevalent in a particular test system. Nevertheless, findings obtained with the aggregometer (11), a commonly used device for the study of platelet function based on the response to exogenous agonists, have favored the prevalent concept that fibrinogen is the essential adhesive protein mediating platelet-to-platelet contact $(12,13)$. In the aggregometer, however, platelet-rich plasma is subjected to shear forces, due to stirring, that differ considerably from those existing in the circulation, so that the possible role of rheological parameters in platelet aggregation cannot be properly evaluated.

Blood circulating in vessels is exposed to shear stress caused by the force necessary to produce flow; the difference in velocity between layers situated at varying distances from the vessel wall determines the shear rate, which is directly proportional to the shear force and inversely proportional to the viscosity of blood. Studies performed under controlled conditions of high shear stress, such to mimic the rheological situation existing in certain districts of the arterial circulation, have suggested that adhesive ligands distinct from fibrinogen may participate in platelet aggregation (14-18). With a series of key observations, Moake et al. $(14,17)$ and Peterson et al. (16) introduced the concept that von Willebrand factor (vWf) may have a crucial role in high shear induced aggregation. Indeed, $\mathrm{vWf}$ binding to the GP Ib-IX complex was shown to be an essential step in the process, whereas the nature of the adhesive protein, or proteins, engaged in the interaction with GP IIb-IIIa, absolutely required for aggregation, could not be determined. Fibrinogen and fibronectin, in addition to vWf, were considered obvious candidates for the role.

In view of these uncertainties, we undertook these studies to identify the adhesive proteins involved in platelet aggrega-

1. Abbreviations used in this paper: $\left[\mathrm{Ca}^{2+}\right]_{0}$, concentration of calcium ions in the extracellular medium; GP, platelet membrane glycoprotein; vWf, von Willebrand factor. 
tion occurring under controlled shear conditions. The results obtained demonstrate that low shear forces induce formation of reversible platelet aggregates dependent on fibrinogen interaction with GP IIb-IIIa; however, this aggregation is detectable only in the presence of low concentrations of ionized calcium in the medium $\left(\left[\mathrm{Ca}^{2+}\right]_{0}\right)$. At higher shear forces, more stable aggregates are formed that require $\mathrm{vWf}$ binding to both GP Ib-IX and GP IIb-IIIa, and are independent of fibrinogen; this aggregation also occurs at physiological levels of $\left[\mathrm{Ca}^{2+}\right]_{0}$. No other adhesive protein appears to be involved in aggregation induced by high shear stress in the absence of exogenous stimulation of platelets, under hemodynamic conditions that may reflect those in stenosed vessels $(19,20)$. These results underline the potential importance of $\mathrm{vWf}$ in acute arterial thrombosis.

\section{Methods}

Patients and controls. All human subjects who participated in these studies were aware of the experimental nature of the research and gave their informed consent, in accordance with the Declaration of Helsinki. Normal controls were staff members of Keio University with no personal or familial history of abnormal bleeding symptoms. The patients with Glanzmann's thrombasthenia, Bernard-Soulier syndrome, afibrinogenemia, and severe von Willebrand disease fulfilled all the accepted criteria for the diagnosis of these disorders. In particular, the two thrombasthenic patients had no measurable platelet membrane GP IIb-IIIa complex; the two patients with Bernard-Soulier syndrome had no measurable platelet membrane GP Ib; the patient with afibrinogenemia had $<10 \mu \mathrm{g} / \mathrm{ml}$ of plasma fibrinogen; and the two patients with severe von Willebrand disease had $<0.1 \%$ of normal vWf plasma levels. All patients and controls denied consuming drugs known to interfere with platelet function for the week preceding the studies.

Modified viscometer for measuring shear-induced platelet aggregation. The cone and plate viscometer used to measure platelet aggregation induced by varying shear stress was similar to the one described previously (21). It consisted essentially of a helium-neon laser light source at $633 \mathrm{~nm}$, a thermostated cone-plate streaming chamber, and a photocounting unit, the output of which, digitized through an appropriate interface, was analyzed and stored in a microcomputer unit. Shear rate $(\gamma)$ was calculated according to the formula $\gamma=6 N / \theta$, where $N$ is the rotational speed of the cone and $\theta$ is the cone angle. In the device used for these studies the cone angle was $1^{\circ}$ and the distance between the cone and the plate was adjusted with an electronic sensor rather than with the mechanical micrometer screw described before (21). This allowed for a more precise and reproducible measurement of the shear forces applied to the samples. Shear stress was calculated multiplying the shear rate by the viscosity of the fluid, assumed to be 1.2 centipoise for all the platelet-rich plasma samples tested. Platelet suspensions exhibited an inverse linear relationship between the single platelet count and the logarithm of transmitted light intensity. This is expressed by the equation $\log \left(I_{\mathrm{t}} / I_{\mathrm{i}}\right)=-k \times l \times c$, where $I_{\mathrm{t}}$ and $I_{\mathrm{i}}$ are the transmitted and incident light intensity, $\mathrm{k}$ is the absorption coefficient, 1 is the effective light path length, and $c$ is the single platelet count. Thus, $\Delta \log I$ expresses the extent of platelet aggregation, namely the decrease in single platelet count, since $\Delta \log I=\log I_{\mathrm{ta}} / I_{\mathrm{i}}-\log I_{\mathrm{tb}} / I_{\mathrm{i}}=k$ $\times l \times\left(C_{\mathrm{b}}-C_{\mathrm{a}}\right)$, where $I_{\mathrm{ta}}$ and $I_{\mathrm{tb}}$ are transmitted light intensities, and $C_{\mathrm{a}}$ and $C_{\mathrm{b}}$ are single platelet counts, after (a) and before (b) application of shear stress, respectively. Changes in the optical density of the platelet suspension corresponded to the occurrence of platelet-to-platelet interactions leading to the formation of aggregates, as shown by electron microscopic analysis (21).

Measurement of platelet aggregation. The anticoagulant used for blood collection was either trisodium citrate $(0.011 \mathrm{M}$ final concentration), which acts to decrease the available concentration of $\mathrm{Ca}^{2+}$ necessary for several reactions that lead to clotting (2), or hirudin (Seikagaku
Kogyo, Tokyo, Japan), a potent and specific thrombin inhibitor obtained from the saliva of the common leech, Hirudo medicinalis. Hirudin was used at the final concentration of $200 \mathrm{U} / \mathrm{ml}$ ( $1 \mathrm{U}$ of hirudin neutralizes the activity of $1 \mathrm{NIH}$ unit of $\alpha$-thrombin). Hirudin rapidly and irreversibly blocks the fibrinogen clotting activity of thrombin (reviewed in 22) but has no effect on the concentration of $\mathrm{Ca}^{2+}$ or other divalent cations. Platelet-rich plasma was prepared by centrifugation at $100 \mathrm{~g}$ for $15 \mathrm{~min}$ at $22-25^{\circ} \mathrm{C}$; the platelet count was adjusted to 3 $\times 10^{5} / \mu$ l using homologous platelet-poor plasma, obtained by centrifugation of blood at $3,000 \mathrm{~g}$ for $20 \mathrm{~min}$. When studying patients with Bernard-Soulier syndrome, who have thrombocytopenia, the platelet count in the control normal platelet-rich plasma was adjusted to the same values obtained in the thrombocytopenic patients, $\sim 1 \times 10^{5} / \mu 1$.

For measuring shear-induced aggregation, $400 \mu \mathrm{l}$ of platelet-rich plasma was applied onto the surface of the polymethylmethacrylate plate and exposed to varying shear stress for $5 \mathrm{~min}$ at $37^{\circ} \mathrm{C}$. Aggregation was monitored continuously by recording the intensity of the light transmitted through the platelet suspension from the beginning of application of the shear forces.

Agonist-induced platelet aggregation was measured in an aggregometer (Chrono-Log Corp., Havertown, PA), as described previously (23). In this case, aggregation was expressed as percent light transmittance, after adjusting the aggregometer at $0 \%$ transmittance with platelet-rich plasma and $100 \%$ transmittance with platelet-poor plasma.

Monoclonal antibodies. All the monoclonal antibodies used in these studies were obtained and characterized as described previously. $\mathrm{LJ}-\mathrm{Ib} 1$ is a murine $\mathrm{IgG}_{1}$ obtained by immunization with purified GP Ib-IX complex (24). This antibody reacts with the amino terminal 45$\mathrm{kD}$ domain of GP Ib $\alpha$ containing the vWf-binding domain $(25,26)$; it inhibits completely vWf binding to platelets mediated by ristocetin (24), but has no effect on the binding to GPIIb-IIIa induced by thrombin. $\mathrm{LJ}-\mathrm{CP} 8$ is a murine $\mathrm{IgG}_{1}$, obtained by immunization with washed human chymotrypsin-treated platelets; it reacts with the GP IIb-IIIa complex (27) and completely blocks the interaction of $\mathrm{vWf}$, fibrinogen, fibronectin, and vitronectin with GP IIb-IIIa. LJ-152B/6 is a murine $\mathrm{IgG}_{1}$ obtained by immunization with a synthetic peptide containing the Arg-Gly-Asp sequence and flanking residues of $\mathrm{vWf}(28)$. This antibody reacts specifically with the GP IIb-IIIa binding site of vWf; has no cross-reactivity with fibrinogen, fibronectin, or vitronectin, even though all these molecules contain at least one Arg-Gly-Asp sequence homologous to that recognized by the antibody in vWf; and selectively inhibits vWf binding to GP IIb-IIIa (28). All IgG were purified on protein-A Sepharose (Sigma Chemical Co., St. Louis, MO) as described (29), then dialyzed against a buffer composed of $20 \mathrm{mM}$ 4-(2-hydroxyethyl)-1-piperazine ethane sulfonic acid (Hepes) and $150 \mathrm{mM} \mathrm{NaCl}, \mathrm{pH}$ 7.4 (Hepes buffer), and stored at $-70^{\circ} \mathrm{C}$ until used.

\section{Results}

Aggregation in platelet-rich plasma containing trisodium citrate as anticoagulant was absent in two patients with Glanzmann's thrombasthenia, i.e., deficient in GP IIb-IIIa, both at low (12 dyn $\left./ \mathrm{cm}^{2}\right)$ and high shear ( $\left.108 \mathrm{dyn} / \mathrm{cm}^{2}\right)$; in contrast, in two patients with Bernard-Soulier syndrome, i.e., deficient in GP Ib-IX, aggregation was normal at low but absent at high shear (Fig. 1). As typically expected in these cases, ADP-induced aggregation was absent in the thrombasthenic patients but normal in the Bernard-Soulier patients (not shown). Contrasting results were also found in one patient with afibrinogenemia, in whom aggregation was absent at low shear but normal at high shear, and in two patients with a complete deficiency of vWf, in whom aggregation was normal at low shear but absent at high shear (Fig. 1). Again, as expected in these cases, ADPinduced aggregation was absent in the afibrinogenemic patient but normal in the two with severe von Willebrand disease (not shown). Note that the extent of aggregation observed at low 


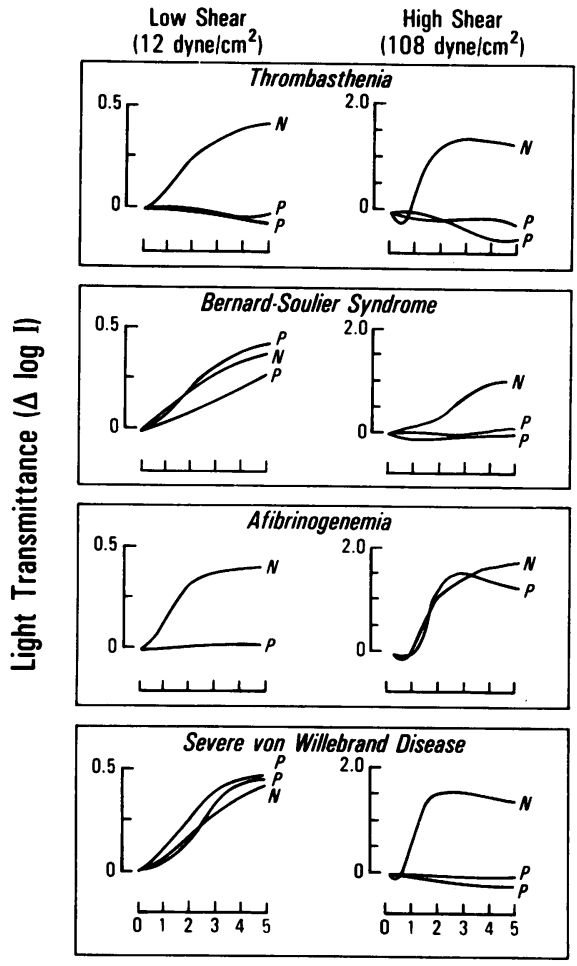

Time (minutes)

Figure 1. Shear-induced aggregation in patients with congenital defects of platelet membrane glycoproteins or plasma adhesive proteins. Platelet-rich plasma, obtained from blood containing $0.011 \mathrm{M}$ trisodium citrate, was exposed to shear forces of 12 or $108 \mathrm{dyn} / \mathrm{cm}^{2}$ for 5 $\min$ at $37^{\circ} \mathrm{C}$. Platelet count was adjusted to $3 \times 10^{8} / \mathrm{ml}$ in all samples, except for the study of Bernard-Soulier patients, in which the count in the control plasma was adjusted to the same value $\left(1 \times 10^{5}\right.$ platelets $/ \mu \mathrm{l})$ obtained in the thrombocytopenic patients. Shear-induced aggregation was monitored by measuring changes in light transmittance through the platelet suspension, expressed as $\Delta \log$ I (see Methods). Note the different scale used on the ordinate for experiments performed at the two different values of shear force. Aggregation was evaluated in two patients with Glanzmann's thrombasthenia (lack of platelet GP IIb-IIIa), two with Bernard-Soulier syndrome (lack of platelet GP Ib-IX), one with afibrinogenemia $(<5 \mu \mathrm{g} / \mathrm{ml}$ plasma fibrinogen concentration), and two with severe (type III) von Willebrand disease ( $\mathrm{vWf}$ concentration $<0.1 \%$ of normal). $\mathrm{N}$ indicates control platelet-rich plasma; $P$, patient platelet-rich plasma. Platelets from the patients with Bernard-Soulier syndrome and thrombasthenia had negligible levels of GP Ib-IX and GP IIb-IIIa complex, respectively, as measured by binding of appropriate monoclonal antibodies. Diagnosis of afibrinogenemia and von Willebrand disease was based on radioimmunological assay of the pertinent plasma protein. Note that at low shear, aggregation was absent in thrombasthenia and afibrinogenemia, while at high shear, aggregation was present only in afibrinogenemia.

shear forces was considerably less than at higher shear forces (compare the scales for $\Delta \log I$ in Fig. 1).

To explore in more detail the effect of shear forces on platelet aggregation, a gradient varying between 6 and $108 \mathrm{dyn} / \mathrm{cm}^{2}$ was applied to citrated platelet-rich plasma over a 5-min period. After an initial $15 \mathrm{~s}$ at $6 \mathrm{dyn} / \mathrm{cm}^{2}$, the gradient varied between 6 and $12 \mathrm{dyn} / \mathrm{cm}^{2}$ in $90 \mathrm{~s}$ (Fig. 2); this resulted in an increase in light transmittance, corresponding to the formation of platelet aggregates as judged by the decrease in single-platelet

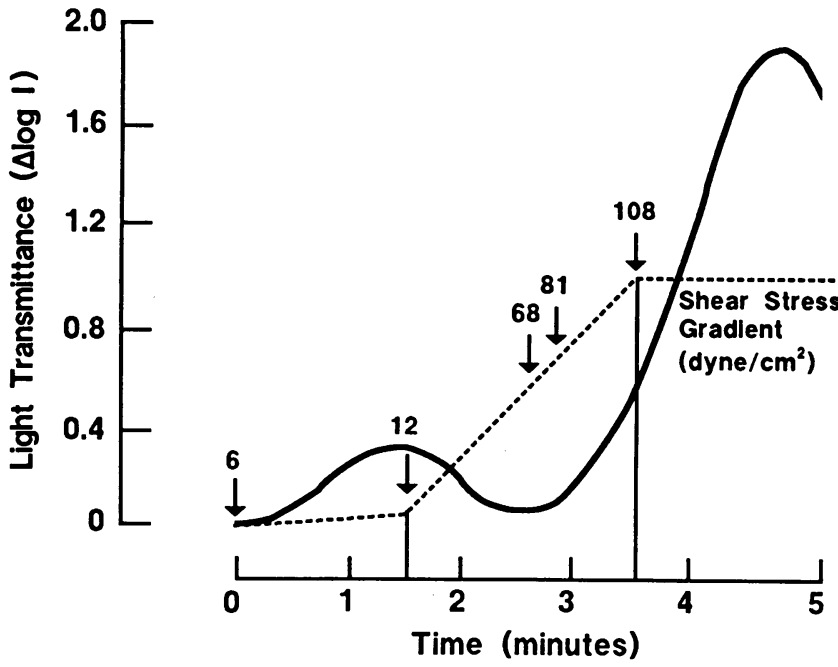

Figure 2. Platelet aggregation induced by varying shear stress. Citrated platelet-rich plasma was exposed to a gradient of $6-108 \mathrm{dyn} / \mathrm{cm}^{2}$ over a 5 -min period at $37^{\circ} \mathrm{C}$. After an initial $15 \mathrm{~s}$ at $6 \mathrm{dyn} / \mathrm{cm}^{2}$ (not shown in the figure), the gradient (indicated by the broken line) varied between 6 and $12 \mathrm{dyn} / \mathrm{cm}^{2}$ in $90 \mathrm{~s}$, between 12 and $108 \mathrm{dyn} /$ $\mathrm{cm}^{2}$ in the next $120 \mathrm{~s}$, and a constant $108 \mathrm{dyn} / \mathrm{cm}^{2}$ in the last $90 \mathrm{~s}$. Aggregation was measured as described in Methods. The value of 68 $\mathrm{dyn} / \mathrm{cm}^{2}$ indicates the point of the gradient at which the initial aggregation was completely reversed. The value of $81 \mathrm{dyn} / \mathrm{cm}^{2}$ indicates the point of the gradient at which a second wave of aggregation initiated.

count, with a peak at $11.5 \pm 1.4 \mathrm{dyn} / \mathrm{cm}^{2}$ (mean $\pm S D$ in 30 normal samples). In the ensuing $120 \mathrm{~s}$, the gradient varied between 12 and $108 \mathrm{dyn} / \mathrm{cm}^{2}$. This resulted initially in a decrease in light transmittance (disaggregation), which returned essentially to the baseline value when the shear gradient reached $68 \pm 11.4$ $\mathrm{dyn} / \mathrm{cm}^{2}$ at $160 \mathrm{~s}$ after the initial application of shear stress, followed by a much more pronounced second increase in light transmittance that started approximately when the shear gradient reached $81 \mathrm{dyn} / \mathrm{cm}^{2}$ at $176 \mathrm{~s}$ (Fig. 2). After the shear force reached $108 \mathrm{dyn} / \mathrm{cm}^{2}$, it was maintained at that level for $90 \mathrm{~s}$, during which a variable decrease in light transmittance was observed. This decrease, presumably reflecting a decrease in the size of aggregates with increase in single platelet count, varied considerably in different individual samples, but light transmittance at the end of $5 \mathrm{~min}$ and under high shear stress was always well above baseline values (Fig. 2).

To confirm the distinct role of different platelet membrane glycoproteins and plasma adhesive proteins in aggregation induced by varying shear stress, the effect of specific monoclonal antibodies was tested at low $\left(12 \mathrm{dyn} / \mathrm{cm}^{2}\right)$ and high shear stress $\left(108 \mathrm{dyn} / \mathrm{cm}^{2}\right)$, as well as in the shear gradient between 6 and $108 \mathrm{dyn} / \mathrm{cm}^{2}$ described above. The aggregation observed when low shear stress was applied to citrated platelet-rich plasma was inhibited only by the anti-GP IIb-IIIa monoclonal antibody LJ-CP8, which completely blocks the interaction of all adhesive proteins with this site; no inhibition was observed either with the anti-GP Ib antibody $\mathrm{LJ}-\mathrm{Ib} 1$, which inhibits $\mathrm{vWf}$ binding to GP Ib, or with the anti-vWf monoclonal antibody $\mathrm{LJ}$ 152B/6, which selectively inhibits binding to GP IIb-IIIa by blocking specifically the function of the Arg-Gly-Asp sequence in $\mathrm{vWf}$ (Fig. 3). These results exclude a role for $\mathrm{vWf}$ in platelet aggregation under low shear force. In contrast, the aggregation 


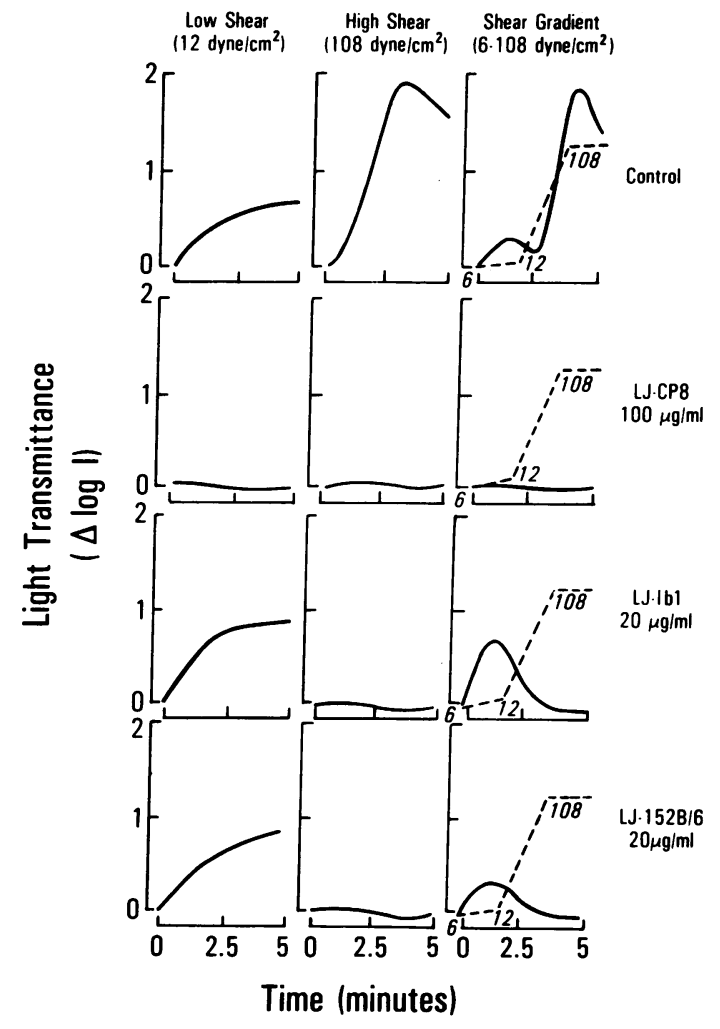

Figure 3. Effect of monoclonal antiplatelet and anti-vWf antibodies on aggregation induced by shear stress. Normal platelet-rich plasma $\left(3 \times 10^{8}\right.$ platelets $\left./ \mathrm{ml}\right)$, obtained from blood collected in the presence of trisodium citrate, was incubated for $5 \mathrm{~min}$ at $22-25^{\circ} \mathrm{C}$ with the anti-GP IIb-IIIa antibody LJ-CP8 $(100 \mu \mathrm{g} / \mathrm{ml})$; or the anti-GP Ib antibody $\mathrm{LJ}-\mathrm{Ibl}(20 \mu \mathrm{g} / \mathrm{ml})$; or the anti-vWf antibody $\mathrm{LJ}-152 \mathrm{~B} / 6(20$ $\mu \mathrm{g} / \mathrm{ml}$ ) that inhibits selectively vWf binding to GP IIb-IIIa but not to GP Ib; or Hepes buffer instead of antibody (control curve). Shearinduced aggregation was measured at $37^{\circ} \mathrm{C}$ for 5 min either at two constant values (see Fig. 1) or at varying shear force (see Fig. 2), as indicated (the broken line in the panels to the right indicates the change in shear force over time). Note that only the anti-GP IIb-IIIa antibody inhibited aggregation induced by low shear force, either applied continuously or in the initial part of the gradient, whereas all three antibodies inhibited high shear-induced aggregation. The concentration used for each antibody was known to give maximal binding to the corresponding target antigen.

induced by high shear stress was inhibited completely by each of the three antibodies (Fig. 3), suggesting that under these conditions vWf-binding to both its platelet receptors, and particularly binding to GP IIb-IIIa through the Arg-Gly-Asp sequence, is necessary for aggregation to occur. The results observed with the shear gradient were concordant with those observed at constant shear stress values, since the first wave of aggregation was inhibited only by the nonselective anti-GP IIbIIIa antibody, while the second was inhibited also by the antiGP Ib antibody and the anti-vWF antibody specifically inhibiting binding to GP IIb-IIIa (Fig. 3).

The experiments reported to this point are compatible with the hypothesis that the binding of vWf to both GP Ib and GP IIb-IIIa is necessary to support aggregation under high shear forces and in the absence of exogenous agonists. Since the direct binding of soluble vWf to GP Ib may be inhibited by $\mathrm{Ca}^{2+}$ ions, as shown previously with asialo VWF (30), shear-induced aggregation was also tested using platelet-rich plasma containing hirudin as anticoagulant, and thus at normal blood concentrations of ionized calcium. These experiments were performed to rule out the possibility that the results described here, as well as those obtained by others $(14,16,17)$, might be artifacts caused by lower than normal divalent cation concentration. Indeed, the aggregation induced by low shear force in plasma containing citrate (Fig. 2) was practically absent in plasma containing hirudin as anticoagulant (Fig. 4). In four separate experiments with normal platelet-rich plasma, the corresponding values of $\Delta \log I( \pm \mathrm{SD})$ were $0.5 \pm 0.1$ in the presence of citrate and $0.1 \pm 0.05$ in its absence. In contrast, the aggregation induced by high shear force was still prominent in the plasma containing hirudin although reduced in extent as compared with that seen in plasma containing citrate (compare Figs. 2 and 4); the corresponding $\Delta \log I$ values were $0.8 \pm 0.3$ and $1.5 \pm 0.4$, respectively. Nevertheless, the inhibitory effect of the anti-GP Ib and anti-GP IIb-IIIa antibodies was identical under the two different experimental conditions (compare Figs. 3 and 4). Results of parallel experiments with the same plasma sample confirmed that the anti-GP IIb-IIIa antibody completely

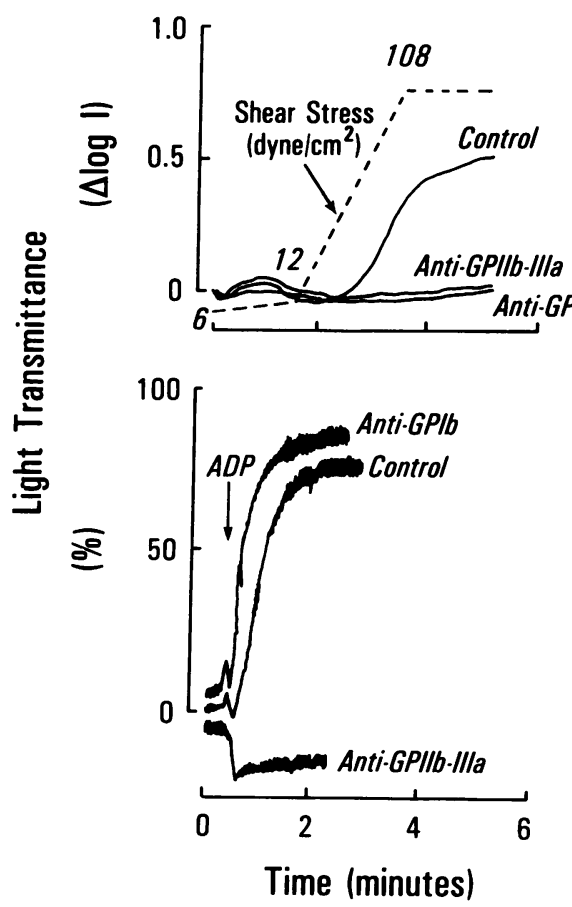

Figure 4. Effect of monoclonal anti-GP Ib and anti-GP IIb-IIIa antibodies on platelet aggregation induced by shear stress or ADP in the presence of physiologic $\left[\mathrm{Ca}^{2+}\right]_{0}$. Normal platelet-rich plasma $\left(3 \times 10^{8}\right.$ platelets $/ \mathrm{ml})$, obtained from blood containing hirudin $(200 \mathrm{U} / \mathrm{ml})$, was incubated for $5 \mathrm{~min}$ at $22-25^{\circ} \mathrm{C}$ with the anti-GP IIb-IIIa antibody LJ-CP8 $(100 \mu \mathrm{g} / \mathrm{ml})$, or the anti-GP Ib antibody LJ-Ib1 $(20 \mu \mathrm{g} /$ $\mathrm{ml}$ ), or Hepes buffer instead of antibody (control). The mixtures were then exposed to varying shear stress (top panel; the broken line indicates the change in shear force over time) or stimulated with 10 $\mu \mathrm{M}$ ADP in the aggregometer (bottom panel), at $37^{\circ} \mathrm{C}$ in either case. Note the absence of significant aggregation at low shear as compared to that seen with citrated plasma (see Figs. 2 and 3); note also that both antibodies blocked aggregation induced by high shear force, but that only the anti-GP IIb-IIIa antibody blocked ADP-induced aggregation. Aggregation was measured as $\Delta \log I$, top panel, or percent light transmittance, bottom panel (see Methods). 
inhibited ADP-induced aggregation as measured in the aggregometer, while the anti-GP Ib antibody had no inhibitory effect (Fig. 4). Aggregation induced by high shear stress in the presence of physiologic ionized calcium concentrations was completely inhibited by the anti-vWf antibody that selectively blocks binding to GP IIb-IIIa but not to GP Ib, while the same antibody had no effect on ADP-induced aggregation measured in the aggregometer (Fig. 5). Because these experiments were performed using normal plasma containing all the circulating adhesive proteins and normal concentrations of divalent cations, the results indicate that $\mathrm{vWf}$ is the only adhesive protein in plasma necessary to support aggregation induced by high shear force, a function requiring interaction with both GP IIbIIIa, through the Arg-Gly-Asp sequence, and GP Ib-IX, through the specific binding site for this receptor.

\section{Discussion}

Our results characterize the distinct adhesive proteins and membrane receptors involved in platelet aggregation occurring under varying shear stress. In the absence of exogenous agonists, aggregation in platelet-rich plasma with low ionized calcium levels (containing $11 \mathrm{mM}$ trisodium citrate) exhibits two distinct phases: one, of relatively small amplitude, with a peak occurring at $\sim 12 \mathrm{dyn} / \mathrm{cm}^{2}$; the other, two- to fourfold greater in magnitude, occurring maximally at above $81 \mathrm{dyn} / \mathrm{cm}^{2}$. The molecular mechanisms mediating platelet-to-platelet contact under varying shear stress are different; fibrinogen interaction with GP IIb-IIIa appears necessary at low shear, while vWf interaction with both GP Ib-IX and GP IIb-IIIa, but independent of fibrinogen, is required at high shear. When platelet-rich plasma contains normal levels of divalent cations, aggregation is negligible at low shear values but conspicuous above $81 \mathrm{dyn} /$ $\mathrm{cm}^{2}$, even though its maximal extent is one-fourth to one-third of that seen in citrated plasma. Under these conditions, platelet-to-platelet contact can only be maintained by vWf binding to both GP Ib and GP IIb-IIIa, as shown concordantly by the results obtained in patients with selective congenital deficiencies of platelet membrane glycoproteins or plasma adhesive proteins, and with the use of specific monoclonal antibodies directed at GP Ib, GP IIb-IIIa, or vWf.

The aggregation induced by low shear stress $\left(12 \mathrm{dyn} / \mathrm{cm}^{2}\right)$ in platelet suspensions with decreased $\left[\mathrm{Ca}^{2+}\right]_{0}$ may not correspond to conditions of pathophysiologic relevance, since it is negligible or absent when divalent cation concentration is within the normal range. This may explain why stable platelet aggregates do not form in the normal circulation, where the time-average range of shear stress in arterial vessels is in the order of $15 \mathrm{dyn} / \mathrm{cm}^{2}$ (17). Regardless of the physiologic relevance, aggregation induced by low shear stress involves the same platelet binding site (GP IIb-IIIa) and plasma adhesive protein (fibrinogen) supporting aggregation induced by exogenous agonists. Since GP IIb-IIIa acquires binding capacity for soluble ligands only after platelet activation $(9,10)$, it appears that exposure to shear force mimics the action of exogenous agonists. Interaction of platelets with the surfaces of the apparatus and/or active release or leakage of granular components have been ruled out in previous studies $(31,32)$, particularly with shear forces below $30 \mathrm{dyn} / \mathrm{cm}^{2}(17,32)$. The possible role of thromboxane $A_{2}$, however, has been suggested by the observation that a thromboxane synthetase inhibitor can block fibrinogen-dependent shear-induced aggregation (32). Indeed, activation of the arachidonate pathway occurs in human platelets brought in close proximity in media with low $\left[\mathrm{Ca}^{2+}\right]_{0}(33)$, and this could explain why aggregation induced by low shear stress was negligible in plasma with normal divalent cation concentration. The exact mechanisms by which thromboxane $\mathrm{A}_{2}$ synthesis may be stimulated by shear stress, however, remain unknown at present. Another possibility is that shear stress affects the conformation and function of GP IIb-IIIa and/or fibrinogen, inducing binding of fibrinogen to unstimulated platelets in the absence of exogenous agonists. For example, it has been shown that fibrinogen insolubilized onto polystyrene beads can interact with GP IIb-IIIa without prior activation of platelets (34), and it is possible that shear stress affects the conformation of fibrinogen in a manner similar to that caused by adsorption onto a surface.

The findings of a previous study (32) suggested that fibrinogen is a necessary cofactor for aggregation induced by shear force values between 54 and $90 \mathrm{dyn} / \mathrm{cm}^{2}$. Our results are in apparent contradiction with those conclusions, since we have now found that aggregation at shear forces above $81 \mathrm{dyn} / \mathrm{cm}^{2}$ is independent of fibrinogen. In the viscometer used for the previous set of experiments, however, the distance between the cone and the plate was adjusted mechanically; thus, variations in this distance may have led to inaccurate estimation of the shear forces applied to the samples. In the modified viscometer used for the experiments described here, the distance was maintained within strict limits with an electronic sensor; thus, the shear forces applied to the samples could be calculated with more precision. However, both this study and the previous study agree that shear-induced aggregation mediated by fibrin-

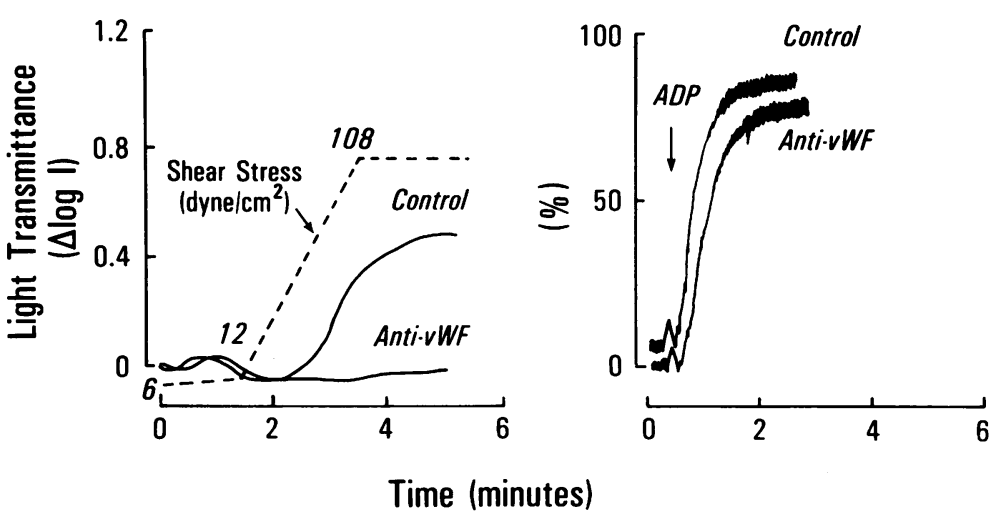

Figure 5. Effect of anti-vWf antibody on platelet aggregation induced by shear stress or ADP in the presence of physiologic $\left[\mathrm{Ca}^{2+}\right]_{0}$. Normal platelet-rich plasma, obtained from blood containing hirudin $(200 \mathrm{U} / \mathrm{ml})$, was incubated for $5 \mathrm{~min}$ at $22-25^{\circ} \mathrm{C}$ with the anti-vWf antibody $\mathrm{LJ}$ $152 \mathrm{~B} / 6(20 \mu \mathrm{g} / \mathrm{ml})$ that selectively blocks $\mathrm{vWf}$ binding to GP IIb-IIla but not to GP Ib; or with Hepes buffer instead of antibody (control curve). The mixtures were then exposed to varying shear stress for $5 \mathrm{~min}$ (left panel; the broken line indicates the change in shear force over time) or stimulated with $10 \mu \mathrm{M}$ ADP in the aggregometer (right panel), at $37^{\circ} \mathrm{C}$ in either case. Aggregation was measured as described in the legend to Fig. 4. Note that this antibody completely blocked aggregation induced by high shear stress but not that induced by ADP. 
ogen leads to the formation of loose aggregates that are reversible when shear forces increase, as shown here, or when exposure to shear stress is terminated, as described previously (32). Difficulties in maintaining a carefully measured shear stress value may also explain the fact that others (17) have observed $v W f-d e p e n d e n t$ aggregation at shear stress values between 30 and $60 \mathrm{dyn} / \mathrm{cm}^{2}$, while we found that it occurs only above 81 $\mathrm{dyn} / \mathrm{cm}^{2}$.

From the results presented here and previously by others $(16,17)$, it is clear that vWf binding to GP Ib is necessary to support platelet aggregation induced by shear forces above 81 dyn $/ \mathrm{cm}^{2}$. In contrast, no conclusive evidence had been obtained to date with regard to the adhesive protein, or proteins, contributing to the process in an essential manner by interacting with GP IIb-IIIa. In fact, the results obtained with congenitally deficient platelets indicated the important role of this binding site but not the nature of the ligand involved, since GP IIb-IIIa can interact with four distinct adhesive proteins, namely vWf $(35)$, fibrinogen $(36,37)$, fibronectin $(38)$, and vitronectin $(39,40)$. The results obtained with anti-GP IIb-IIIa antibodies were inconclusive for similar reasons, since the one used here (LJ-CP8), as well as those used in previous studies $(16,17)$, inhibit nonselectively the binding function of GP IIbIIIa. Finally, the demonstration that shear-induced aggregation of washed platelets was apparent only after addition of $\mathrm{vWf}$ and not fibrinogen (17) cannot be taken to prove that, in the presence of all plasma proteins, the process is actually mediated by vWf binding to GP IIb-IIla. Indeed, as shown for agonist-induced aggregation (23), vWf might exhibit a functional role as a GP IIb-IIIa ligand only in the absence of fibrinogen, but have no activity of this kind when the latter is present (41-43). The necessary presence of $\mathrm{vWf}$ for the occurrence of aggregation in a purified system, as well as in plasma, could simply reflect the absolute requirement for its interaction with GP Ib, a function that cannot be substituted for by another protein the way $\mathrm{vWf}$ can substitute for fibrinogen in binding to GP IIb-IIIa.

The experiments presented here, however, demonstrate conclusively that the interaction of vWf with GP IIb-IIIa, as well as with GP Ib, is the event necessary and sufficient to support aggregation induced by high shear stress. In fact, an antibody selectively inhibiting vWf binding to GP IIb-IIIa (28) blocked shear-induced aggregation but not ADP-induced aggregation. Since this antibody recognizes specifically a vWf epitope including the Arg-Gly-Asp sequence (28), its inhibitory effect clearly proves the biological relevance of this adhesive site in vWf. Moreover, in view of the fact that these experiments were performed with platelet-rich plasma containing the full complement of plasma proteins at their physiologic concentrations, it is clear that vWf interaction with GP IIb-IIIa, mediated by the Arg-Gly-Asp sequence (28), can occur in the presence of normal plasma levels of the other GP IIb-IIIa ligands containing the same adhesion sequence, in particular fibronectin and fibrinogen. This conclusion is in contrast to previous suggestions (41-43), based essentially on a consideration of the significantly lower concentration of $\mathrm{vWf}$ relative to the aforementioned adhesive proteins in plasma. While it appears that vWf binding to GP IIb-IIIa is essential for mediating stable interplatelet contact during shear stress, it remains to be demonstrated whether, in high shear conditions, fibrinogen is excluded from binding to GP IIb-IIIa, or functionally inefficient even if bound. The ability of the multivalent $\mathrm{vWf}$ protein to interact with several binding sites on adjacent platelets seems ideally suited to maintain intercellular contacts under high shear stress, a role that fibrinogen may perform under less rigorous flow conditions.

Experimental studies of platelet aggregation, like those reported here, are usually based on measuring the occurrence of interplatelet contacts in suspension. On the contrary, the process of thrombus growth in the intact organisms involves platelet adhesion to exposed subendothelium followed by "aggregation" of incoming platelets to the adhering ones to form the thrombus mass (2). Thus, during thrombogenesis in vivo, platelets become immobilized onto a surface rather than aggregating in suspension. Under high shear stress conditions, attachment of platelets to surface-bound vWf through GP Ib-IX is crucial for initial adhesion (44). Our results suggest that this interaction, even in the absence of other agonists or activation of clotting, may trigger a platelet response leading to expression of the binding function of GP IIb-IIIa which, by binding vWf from the surrounding medium, can strengthen the interplatelet contact to sustain the opposing effect of shear forces. One can envision a mechanism by which the interplatelet interaction leading to thrombus growth is initially GP Ib-dependent, but becomes progressively reinforced by expression of the binding function of GP IIb-IIIa. This hypothesis of a self-sustaining process involving two adhesion receptors and one adhesive ligand is consistent with the experimental findings, presented here and previously by others $(16,17)$, that a block in vWf binding to either GP Ib-IX or GP IIb-IIIa results in obliteration of shear-induced aggregation. This hypothesis also implies that, under the particular conditions of high shear stress, platelet activation can follow the initial attachment mediated by $\mathrm{vWf}$ and GP Ib. Indeed, vWf interaction with GP Ib has been shown to induce, in the absence of any other exogenous agonists, functional activity of the GP IIb-IIIa receptor which, in turn, can support platelet aggregation $(30,45)$. It remains to be understood how shear force above a threshold value can promote the initial binding of vWf to GP Ib, possibly through conformational changes of components of the platelet surface (16), and how this can affect the binding function and/or selectivity of GP IIb-IIIa.

With regard to acute arterial occlusion, platelets in circulating blood may become exposed to shear stress values in excess of several hundred dyn $/ \mathrm{cm}^{2}$, as in small arteries and arterioles partially obstructed by atherosclerotic processes or as a consequence of vasospasm $(19,20)$. If, as suggested by our findings, vWf binding to GP Ib-IX and GP IIb-IIIa becomes crucial in initiating and mediating the formation of occlusive thrombi in stenosed atherosclerotic vessels, strategies directed at inhibiting these interactions may be useful in preventing thrombotic vascular occlusion. This approach may also provide selectivity and result in lesser antihemostatic consequences, if other adhesive proteins, such as fibrinogen, support hemostatic plug formation at lower shear stress and/or in the presence of other platelet agonists.

\section{Acknowledgments}

We wish to thank James R. Roberts and M. Lisa Thorn for their work in the preparation and characterization of the monoclonal antibodies; Keiko Kawakami and Mayumi Fukuyama for their excellent technical assistance; Eileen Bristow for her excellent secretarial assistance; and Marina Hoffman for her help in the preparation of the manuscript.

This work was supported in part by National Institutes of Health 
grants HL-31950 and HL-15491, and by grant RR0833 to the General Clinical Research Center of Scripps Clinic. This is publication No. 6330/CVB from the Research Institute of Scripps Clinic.

\section{References}

1. Weiss, H. J. 1975. Platelet physiology and abnormalities of platelet function. N. Engl. J. Med. 293:580-588.

2. Sixma, J. J., and J. Wester. 1977. The hemostatic plug. Semin. Hematol. 14:265-299.

3. Chapmann, I. 1965. Morphogenesis of occluding coronary artery thrombosis. Arch. Pathol. 80:256-261.

4. Falk, E. 1985. Unstable angina with fatal outcome: dynamic coronary thrombosis leading to infarction and/or sudden death: autopsy evidence of recurrent mural thrombosis with peripheral embolization culminating in total vascular occlusion. Circulation. 71:699-708.

5. Fuster, V., P. M. Steele, and J. H. Chesebro. 1985. Role of platelets and thrombosis in coronary atherosclerotic disease and sudden death. J. Am. Coll. Cardiol. 5:175B-84B.

6. Davies, M. J., A. C. Thomas, P. A. Knapman, and J. R. Hangartner. 1986. Intramyocardial platelet aggregation in patients with unstable angina suffering sudden ischemic cardiac death. Circulation. 73:418-427.

7. Fitzgerald, D. J., L. Roy, F. Catella, and G. A. Fitzgerald. 1986. Platelet activation in unstable coronary disease. $N$. Engl. J. Med. 315:983-989.

8. Packham, M. A., and J. F. Mustard. 1984. Progress in Hemostasis and Thrombosis. Vol. 7. Platelet Adhesion. T. H. Spaet, editor. Grune \& Stratton, Inc., Orlando, FL. 211-288.

9. Kroll, M. H.,and A. I. Schafer. 1989. Biochemical mechanisms of platelet activation. Blood. 74:1181-1195.

10. Phillips, D. R., I. F. Charo, L. V. Parise, and L. A. Fitzgerald. 1988. The platelet membrane glycoprotein IIb-IIIa complex. Blood. 71:831-843.

11. Born, G. V. R. 1962. Quantitative investigations into the aggregation of blood platelets. J. Physiol. (Lond.). 162:67P-68P.

12. Mustard, J. F., R. L. Kinlough-Rathbone, M. A. Packham, D. W. Perry, E. J. Harfenist, and K. R. M. Pai. 1979. Comparison of fibrinogen association with normal and thrombasthenic platelets on exposure to ADP or chymotrypsin Blood. 54:987-993.

13. Marguerie, G. A., E. F. Plow, and T. S. Edgington. 1979. Human platelets possess an inducible and saturable receptor specific for fibrinogen. J. Biol. Chem 254:5357-5363.

14. Moake, J. L., N. A. Turner, N. A. Stathopoulos, L. H. Nolasco, and J. D. Hellums. 1986. Involvement of large plasma von Willebrand factor (vWF) multimers and unusually large vWF forms derived from endothelial cells in shear stress-induced platelet aggregation. J. Clin. Invest. 78:1456-1461.

15. O'Brien, J. R., and G. P. Salmon. 1987. Shear stress activation of platelet glycoprotein IIb/IIIa plus von Willebrand factor causes aggregation:filter blockage and the long bleeding time in von Willebrands disease. Blood. 70:1354-1361

16. Peterson, D. M., N. A. Stathopoulos, T. D. Giorgio, J. D. Hellums, and J. L. Moake. 1987. Shear-induced platelet aggregation requires von Willebrand factor and platelet membrane glycoproteins Ib and IIb-IIIa. Blood. 69:625-628.

17. Moake, J. L., N. A. Turner, N. A. Stathopoulos, L. Nolasco, and J. D. Hellums. 1988. Shear-induced platelet aggregation can be mediated by vWF released from platelets, as well as by exogenous large or unusually large vWF multimers, requires adenosine diphosphate, and is resistant to aspirin. Blood. $71: 1366-1374$

18. Weiss, H. J., J. Hawiger, Z. M. Ruggeri, V. T. Turitto, P. Thiagarajan, and T. Hoffmann. 1989. Fibrinogen-independent platelet adhesion and thrombus formation on subendothelium mediated by glycoprotein IIb-IIIa complex at high shear rate. J. Clin. Invest. 83:288-297.

19. Back, C. H., J. R. Radbill, and D. W. Crawford. 1977. Analysis of pulsatile viscous blood flow through diseased coronary arteries of man. J. Biomech. 10:339-353.

20. Lipowski, H. H., S. Usani, and S. Chien. 1980. In vivo measurements of "apparent viscosity" and microvessel hematocrit in the mesentery of the cat. Microvasc. Res. 19:297-319.

21. Fukuyama, M., K. Sakai, I. Itagaki, K. Kawano, M. Murata, Y. Kawai, K. Watanabe, M. Handa, and Y. Ikeda. 1989. Continuous measurement of shear-induced platelet aggregation. Thromb. Res. 54:253-260.

22. Johnson, P. H., P. Sze, R. Winant, P. W. Payne, and J. B. Lazar. 1989. Biochemistry and genetic engineering of hirudin. Semin. Thromb. Hemostasis. 15:302-315.

23. De Marco, L., A. Girolami, T. S. Zimmerman, and Z. M. Ruggeri. 1986. von Willebrand factor interaction with the glycoprotein IIb/IIIa complex. Its role in platelet function as demonstrated in patients with congenital afibrinogenemia. J. Clin. Invest. 77:1272-1277.

24. Handa, M., K. Titani, L. Z. Holland, J. R. Roberts, and Z. M. Ruggeri. 1986. The von Willebrand factor-binding domain of platelet membrane glycoprotein Ib. Characterization by monoclonal antibodies and partial amino acid sequence analysis of proteolytic fragments. J. Biol. Chem. 261:12579-12585.

25. Vicente, V., P. J. Kostel, and Z. M. Ruggeri. 1988. Isolation and functional characterization of the von Willebrand factor-binding domain located between residues $\mathrm{His}(1)-\mathrm{Arg}(293)$ of the alpha-chain of glycoprotein Ib. J. Biol. Chem. 263:18473-18479.

26. Vicente, V., R. A. Houghten, and Z. M. Ruggeri. 1990. Identification of a site in the alpha chain of platelet glycoprotein Ib that participates in von Willebrand factor binding. J. Biol. Chem. 265:274-280.

27. Niiya, K., E. Hodson, R. Bader, V. Byers-Ward, J. A. Koziol, E. F. Plow, and Z. M. Ruggeri. 1987. Increased surface expression of the membrane glycoprotein IIb/IIIa complex induced by platelet activation. Relationship to the binding of fibrinogen and platelet aggregation. Blood. 70:475-483.

28. Berliner, S., K. Niiya, J. R. Roberts, R. A. Houghten, and Z. M. Ruggeri. 1988. Generation and characterization of peptide-specific antibodies that inhibit von Willebrand factor binding to GP IIb-IIIa without interacting with other adhesive molecules: selectivity is conferred by Pro 1743 and other amino acid residues adjacent to the sequence Arg1744-Gly1745-Asp1746. J. Biol. Chem. 263:75007505

29. Ey, P. L., S. J. Prowse, and C. R. Jenkin. 1978. Isolation of pure IgG1, IgG2a and IgG2b immunoglobulins from mouse serum using protein A-Sepharose. Immunochemistry. 15:429-436.

30. De Marco, L., M. Mazzuccato, M. G. Del Ben, U. Budde, A. B. Federici A. Girolami, and Z. M. Ruggeri. 1987. Type IIB von Willebrand factor with normal sialic acid content induces platelet aggregation in the absence of ristocetin. Role of platelet activation, fibrinogen, and two distinct membrane receptors. J. Clin. Invest. 80:475-482.

31. Belval, T. K., J. D. Hellums, and R. T. Solis. 1984. The kinetics of platelet aggregation induced by fluid-shearing stress. Microvasc. Res. 28:279-288.

32. Ikeda, Y., M. Murata, Y. Araki, K. Watanabe, Y. Ando, I. Itagaki, Y. Mori, M. Ichitani, and K. Sakai. 1988. Importance of fibrinogen and platelet membrane glycoprotein IIb-IIla in shear-induced platelet aggregation. Thromb. Res. 51:157-163.

33. Guccione, M. A., M. A. Packham, R. L. Kinlough-Rathbone, D. W. Perry, and J. F. Mustard. 1976. Reactions of polylysine with human platelets in plasma and in suspensions of washed platelets. Thromb. Haemostasis. 36:360 375.

34. Coller, B. S. 1980. Interaction of normal, thrombasthenic, and BernardSoulier platelets with immobilized fibrinogen: defective platelet-fibrinogen interaction in thrombasthenia. Blood. 55:169-178.

35. Ruggeri, Z. M., L. De Marco, L. Gatti, R. Bader, and R. R. Montgomery. 1983. Platelets have more than one binding site for von Willebrand factor. J. Clin. Invest. 72:1-12.

36. Bennett, J. S., G. Vilaire, and D. B. Cines. 1982. Identification of the fibrinogen receptor on human platelets by photoaffinity labeling. J. Biol. Chem. 257:8049-8064

37. Nachman, R. L., and L. L. K. Leung. 1982. Complex formation of platele membrane glycoproteins IIb and IIla with fibrinogen. J. Clin. Invest. 69:263-269.

38. Ginsberg, M. H., J. Forsyth, A. Lightsey, J. Chediak, and E. F. Plow. 1983 Reduced surface expression and binding of fibronectin by thrombin-stimulated thrombasthenic platelets. J. Clin. Invest. 71:619-624.

39. Thiagarajan, P., and K. L. Kelly. 1988. Exposure of binding sites fo vitronectin on platelets following stimulation. J. Biol. Chem. 263:3035-3038.

40. Asch, E., and E. Podack. 1990. Vitronectin binds to activated human platelets and plays a role in platelet aggregation. J. Clin. Invest. 85:1372-1378.

41. Pietu, G., G. Cherel, G. Marguerie, and D. Meyer. 1984. Inhibition of von Willebrand factor-platelet interaction by fibrinogen. Nature (Lond.). 308:648649.

42. Schullek, J., J. Jordan, and R. R. Montgomery. 1984. Interaction of von Willebrand factor with human platelets in the plasma milieu. J. Clin. Invest. 73:421-428.

43. Gralnick, H. R., S. B. Williams, and B. S. Coller. 1984. Fibrinogen competes with von Willebrand factor for binding to the glycoprotein IIb/IIIa complex when platelets are stimulated with thrombin. Blood. 64:797-800.

44. Weiss, H. J., V. T. Turitto, and H. R. Baumgartner. 1978. Effect of shear rate on platelet interaction with subendothelium in citrated and native blood. I. Shear rate-dependent decrease of adhesion in von Willebrand's disease and the Bernard-Soulier syndrome. J. Lab. Clin. Med. 92:750-764.

45. De Marco, L., A. Girolami, S. Russell, and Z. M. Ruggeri. 1985. Interaction of asialo von Willebrand factor with glycoprotein Ib induces fibrinogen binding to the glycoprotein IIb/IIIa complex and mediates platelet aggregation. $J$. Clin. Invest. 75:1 198-1203. 